\title{
Switching a Polar Metal via Strain Gradients
}

\author{
Asier Zabalo $\oplus^{1}$ and Massimiliano Stengel ${ }^{1,2}$ \\ ${ }^{1}$ Institut de Ciència de Materials de Barcelona (ICMAB-CSIC), Campus UAB, 08193 Bellaterra, Spain \\ ${ }^{2}$ ICREA-Institució Catalana de Recerca i Estudis Avanats, 08010 Barcelona, Spain
}

(Received 22 June 2020; accepted 15 February 2021; published 23 March 2021)

\begin{abstract}
Although rare, spontaneous breakdown of inversion symmetry sometimes occurs in a material which is metallic: these are commonly known as polar metals or ferroelectric metals. Their polarization, however, is difficult to switch via an electric field, which limits the experimental control over band topology. Here we investigate, via first-principles theory, flexoelectricity as a possible way around this obstacle with the wellknown polar metal $\mathrm{LiOsO}_{3}$. The flexocoupling coefficients are computed for this metal with high accuracy with an approach based on real-space sums of the interatomic force constants. A Landau-GinzburgDevonshire-type first-principles Hamiltonian is built and a critical bending radius to switch the material is estimated, whose order of magnitude is comparable to that of $\mathrm{BaTiO}_{3}$.
\end{abstract}

DOI: 10.1103/PhysRevLett.126.127601

The so-called polar or ferroelectric metals [1], first proposed by Anderson more than half a century ago in the context of martensitic transformations [2], have been attracting increasing attention recently. Their interest lies on the unusual physics that may emerge from the coexistence of metallicity and polarity, two properties that were initially regarded as contraindicated. For instance, they provide excellent opportunities to study exotic quantum phenomena, like noncentrosymmetric superconductivity $[3,4]$ or spin-polarized currents [5].

The prototypical (and historically the first experimentally known) material realization is lithium osmate, which undergoes a ferroelectriclike transition at $140 \mathrm{~K}$ from the centrosymmetric $R \overline{3} c$ to the noncentrosymmetric $R 3 c$ space group [6]. Since its discovery, the list of known polar metals has been steadily growing [7]. A range of materials-design strategies were successfully demonstrated, including crystal chemistry [8], couplings to antiferrodistortive oxygen tilts [1], interface [1,9] or superlattice $[10,11]$ geometries, or high-throughput computational searches [12]. Progress in characterization techniques has been considerable, too [13].

A long-standing issue for both fundamental research and potential applications (e.g., in nanoscale electronic and thermoelectric devices [14]) concerns the ability to control polarity via an appropriate external field. Because of the presence of free carriers in the bulk, the most obvious means of switching polarity in ferroelectrics, i.e., an applied electric field, is apparently ruled out. Indeed, while electrical switching of thin two-dimensional [15] metals has been demonstrated, a general method for reversing polarity at the bulk level is still missing. Our goal is to demonstrate that flexoelectricity can solve this issue.

Flexoelectricity describes the coupling between a strain gradient and the macroscopic polarization and, unlike its homogeneous counterpart (piezoelectricity), it does not require any particular space group to be present [16-18]. While flexoelectricity is hardly a new discovery [19], its practical relevance was demonstrated only recently, thus reviving this field from both the experimental [20-22] and theoretical [23] points of view. Of course, the electrical polarization can only be defined in insulating crystals [24], so the macroscopic flexoelectric coefficient of a polar metal vanishes identically. However, as we shall demonstrate shortly, the flexocoupling between polar lattice modes and a strain gradient does exist even in metals. Since elastic fields, unlike electric fields, are not screened by free carriers, this constitutes, in principle, a viable means of controlling polarity. Still, whether or not the relevant couplings are strong enough for such a mechanism to be experimentally accessible is currently unknown. First-principles calculations could be very helpful in this context, and indeed electronic-structure methods to study flexoelectricity have seen impressive progress in recent years [23,25-29]. However, the code implementation $[23,30]$ of these techniques is currently limited to insulators; their generalization to metals, while in principle possible, would require substantial analytical derivations and programming, which we regard as unpractical for the scope of the present work.

Here we overcome such limitations by developing an alternative method to calculate flexocoupling coefficients in metals, which is based on real-space sums of the interatomic force constants (IFCs). We demonstrate our computational strategy by calculating the flexocoupling coefficients of $\mathrm{LiOsO}_{3}$ as a test case. We use the aforementioned values, in combination with a first-principlesbased effective Hamiltonian that we have constructed by expanding the energy around the centrosymmetric cubic phase, to estimate the critical bending radius $R_{\text {crit }}$ of $\mathrm{LiOsO}_{3}$. For comparison purposes, we perform an 


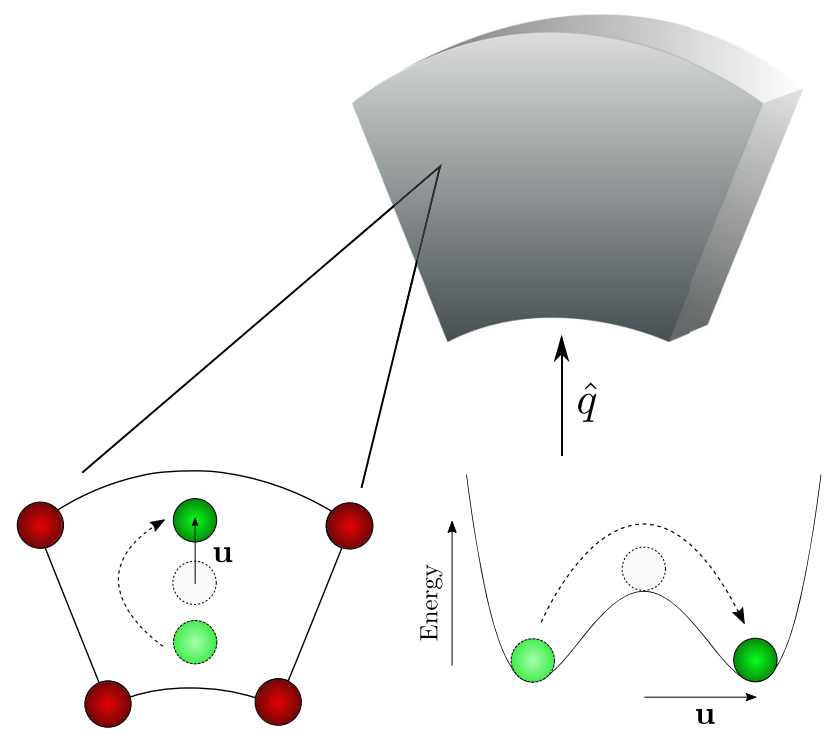

FIG. 1. A bending-type strain gradient is applied to a macroscopic crystal along the direction $\hat{q}$. The external strain gradient couples to the polar modes resulting in a displacement of the atoms and, as a consequence, the structure evolves to another symmetrically equivalent ferroelectric state.

analogous study on $\mathrm{BaTiO}_{3}$, probably one of the most studied ferroelectric compounds. We find a comparable value of $R_{\text {crit }}$ for both materials. Since flexoelectric switching of the polar domains has been experimentally demonstrated [22] in $\mathrm{BaTiO}_{3}$ already, this result indicates that mechanical switching of $\mathrm{LiOsO}_{3}$ mediated by flexoelectricity should be well within experimental reach.

To start with, we consider a setup as illustrated in Fig. 1, i.e., of a $\mathrm{LiOsO}_{3}$ (or $n$-doped $\mathrm{BaTiO}_{3}$ ) sample that is cut along some crystallographic direction $\hat{q}$ and mechanically bent via some external load. Within the interior of the film, the polar order parameter is assumed to be homogeneous, and its amplitude is described by some three-dimensional vector $\mathbf{u}$ with the physical dimension of length. In the following we quantify the coercive bending radius, i.e., the radius of curvature that needs to be applied in order to switch the polar order parameter between two neighboring local minima, which are degenerate at mechanical equilibrium. We shall calculate the critical radius via the following formula,

$$
R_{\text {crit }}=\frac{f_{\text {eff }}}{F_{\text {coerc }}}
$$

where $f_{\text {eff }}$ is the effective flexocoupling coefficient associated with the flexural deformation and $F_{\text {coerc }}$ is the minimal generalized force that is required for the mode $\mathbf{u}$ to cross the energy barrier between two minima. Thus, the problem can be divided into two separate tasks: (i) determining the coupling between a flexural deformation and the polar mode, described by $f_{\text {eff }}$, as a function of the crystallographic orientation, and (ii) identifying the most likely switching paths and the corresponding energetics.

From now on, we shall assume a Landau-like expansion of the energy around the high-symmetry cubic structure as a function of the relevant parameters, following the established common practice in theoretical studies of perovskite ferroelectrics. In this context, task (ii) entails no conceptual difficulties, as it consists in mapping the potential energy surface of the crystal as a function of the relevant lattice degrees of freedom - such a procedure has been successfully carried out for a wide range of materials already [31,32]. The main technical obstacle resides in (i), since no established methods exist for the calculation of $f_{\text {eff }}$ in metals. Given the novelty, we focus on this point in the following. (Further details can be found in Ref. [33], Sec. V.)

In full generality, the flexocoupling tensor in a "softmode" material can be defined as follows [25,27],

$$
f_{\alpha \lambda, \beta \gamma}=\left\langle P_{\alpha} \mid C_{\beta \gamma, \lambda}\right\rangle,
$$

where $\left|C_{\beta \gamma, \lambda}\right\rangle$ describes the forces induced on individual atoms by a gradient along the Cartesian direction $r_{\lambda}$ of the symmetric strain tensor $\varepsilon_{\beta \gamma}$ ("type-II" representation of the strain gradient [25]), while $\left|P_{\alpha}\right\rangle$ represents the (normalized) atomic distortion pattern associated with the ferroelectric mode. (Bras and kets are real vectors of dimension $3 N$, where $N$ is the number of basis atoms; their scalar product stands for a sum over sublattices and Cartesian directions.) The main technical challenge from a computational point of view consists in calculating the flexoelectric force-response tensor $|\mathbf{C}\rangle$. Very recent developments $[23,30]$ in linearresponse theory allow us, in principle, to access this quantity via a long-wave expansion of the dynamical matrix to second order in the wave vector q. Such methodology, however, has not been generalized to metals yet.

To circumvent this obstacle, we use an alternative definition of $|\mathbf{C}\rangle$ that is based on the real-space moments of the interatomic force constants [16,25]. The latter are defined as the second derivative of the total energy with respect to atomic displacements:

$$
\Phi_{\kappa \alpha, \kappa^{\prime} \beta}^{l}=\frac{\partial^{2} E}{\partial u_{\kappa \alpha}^{0} \partial u_{\kappa^{\prime} \beta}^{l}} .
$$

Then, we can write the flexoelectric force-response tensor as [25]

$$
\begin{aligned}
\left|C_{\beta \gamma, \lambda}\right\rangle & =\left|T_{\beta, \gamma \lambda}\right\rangle+\left|T_{\gamma, \lambda \beta}\right\rangle-\left|T_{\lambda, \beta \gamma}\right\rangle, \\
\left\langle\kappa \alpha \mid T_{\beta, \gamma \lambda}\right\rangle & =-\frac{1}{2} \sum_{l \kappa^{\prime}} \Phi_{\kappa \alpha, \kappa^{\prime} \beta}^{l}\left(\mathbf{d}_{\kappa \kappa^{\prime}}^{l}\right)_{\gamma}\left(\mathbf{d}_{\kappa \kappa^{\prime}}^{l}\right)_{\lambda} .
\end{aligned}
$$

Here $\mathbf{d}_{\kappa \kappa^{\prime}}^{l}=\mathbf{R}^{l}+\boldsymbol{\tau}_{\kappa^{\prime}}-\boldsymbol{\tau}_{\kappa}$, where $\mathbf{R}^{l}$ is the Bravais lattice vector of the $l$ th cell and $\tau_{\kappa}$ is the position of atom $\kappa$ within 
the unit cell $l=0 ;|\kappa \alpha\rangle$ is a normalized projector on a given sublattice $\kappa$ and displacement direction $\alpha$. Thus, $\left\langle\kappa \alpha \mid T_{\beta, \gamma \lambda}\right\rangle$ describes the $\alpha$ component of the atomic force produced on atom $\kappa$ by the second gradient of the displacement field. The latter is commonly referred to as "type-I" representation of the strain-gradient tensor [25]; Eq. (4) converts then $|\mathbf{T}\rangle$ into its type-II counterpart $|\mathbf{C}\rangle$.

The lattice sums in Eq. (5) require a sufficiently fast decay of the IFCs as a function of $\left|\mathbf{d}_{\kappa \kappa^{\prime}}^{l}\right|$ to be well defined. This condition is clearly violated in insulators, where the long-ranged nature of the electrostatic forces makes the sums only conditionally convergent [37]. In metals, Kohn anomalies may produce potentially problematic longranged forces as well; however, the assumption of a finite electronic temperature guarantees in practice that the IFCs are short-ranged. This means that the lattice sums of Eq. (5) will eventually converge to the correct physical value when a dense enough q-point mesh is used to calculate the realspace force constants of Eq. (3). This also implies that, unlike in insulators, the flexocoupling tensor in metals is a well-defined bulk property, and it is not altered by boundary effects, such as surface piezoelectricity [3840]. Interestingly, the flexoelectric force-response tensor is directly related (in a crystal that is free of stresses) to the elastic tensor components via [41]

$$
C_{\alpha \lambda, \beta \gamma}=\frac{1}{\Omega} \sum_{\kappa}\left\langle\kappa \alpha \mid C_{\beta \gamma, \lambda}\right\rangle,
$$

where $\Omega$ is the volume of the unit cell. This is a useful consistency check: one can then compare the results with a more conventional calculation of the elastic tensor [42] to gauge the reliability of the flexocoupling coefficients as determined via Eq. (2). Note that the elastic tensor components are themselves a crucial ingredient for calculating the effective flexocoupling of Eq. (1) starting from the flexocoupling tensor $\mathbf{f}$; therefore, it is important to ensure that the two physical quantities are calculated with consistent accuracy.

Our first-principles calculations are performed with the open-source ABINIT $[43,44]$ package. (Details of the computational parameters are provided in Ref. [33], Sec. I.) Numerical results for both $\mathrm{BaTiO}_{3}$ and $\mathrm{LiOsO}_{3}$ are shown in Table I. Clearly, the largest flexocouplings are $f_{12}$ in $\mathrm{LiOsO}_{3}$ and $f_{11}$ for $\mathrm{BaTiO}_{3}$. (The latter material behaves very similarly to $\mathrm{SrTiO}_{3}$ [27], which is natural to expect given the affinities in the electronic and atomic structure.) Their absolute values are similar overall, which provides a first indication that the flexocoupling is comparably strong in these two materials. Note that the discrepancy in the elastic constants calculated via the two different methods is less than $1 \%$ for the three independent components of $\mathrm{LiOsO}_{3}$, which confirms the excellent quality of the calculations. We also show in Fig. S4 (and Table S4) the convergence of the numerical results for both the elastic
TABLE I. Independent components of the calculated elastic (in $\mathrm{GPa}$ ) and flexocoupling (in eV) tensor. "Lattice sums" refers to Eq. (4,5); "DFPT" to the method of Ref. [42]. The $n$-type flexocoupling coefficients [27] of $\mathrm{BaTiO}_{3}$ are shown.

\begin{tabular}{llcccccc}
\hline \hline & & $C_{11}$ & $C_{12}$ & $C_{44}$ & $f_{11}$ & $f_{12}$ & $f_{44}$ \\
\hline \multirow{2}{*}{$\mathrm{LiOsO}_{3}$} & Lattice sums & 364.7 & 129.5 & $44.3-13.8$ & 49.3 & 3.3 \\
& DFPT & 365.6 & 129.5 & 44.1 & $\cdots$ & $\cdots$ & $\cdots$ \\
$\mathrm{BaTiO}_{3}$ & Lattice sums & 346.1 & 121.7 & 134.5 & -53.5 & 3.4 & -39.5 \\
& DFPT & 353.3 & 121.7 & 137.7 & $\cdots$ & $\cdots$ & $\cdots$ \\
\hline \hline
\end{tabular}

and flexocoupling constants as a function of the q-point mesh, further corroborating this point (see Sec. IV of Ref. [33]).

To make further progress, we use the values in Table I to compute the effective flexocoupling coefficients for three representative orientations of the sample ([100], [110], and [111]), either in the beam-bending or the plate-bending limit. (We focus on the beam-bending limit following the definitions of Ref. [45]; explicit formulas are reported in Ref. [33], Sec. IV.) The results, shown in Table II, indicate that [100] is by far the bending direction that produces the largest flexocoupling in $\mathrm{LiOsO}_{3}$. The situation in $\mathrm{BaTiO}_{3}$ seems to be more balanced overall, with a slight preference for [110] and [111] directions over [100]. Note, however, that for each surface orientation $\hat{\mathbf{q}}$, the effective flexocoupling describes the flexoinduced force acting on the polar mode along $\hat{\mathbf{q}}$. Depending on the switching path, such force might not be parallel to the direction along which the polar mode evolves during switching $\hat{\mathbf{s}}$; in such cases the effective flexocoupling needs to be scaled by the projection $\hat{\mathbf{q}} \cdot \hat{\mathbf{s}}$. Since the relevant paths in $\mathrm{BaTiO}_{3}$ (see next paragraph) involve [100]-oriented switching, such geometrical factor reduces the [110] and [111] coefficients by $\sqrt{2}$ and $\sqrt{3}$, respectively, bringing all three values of $f_{\text {eff }}$ to a similar magnitude.

Having calculated the values of $f_{\text {eff }}$, we now need the information about the switching path to obtain $R_{\text {crit }}$ according to Eq. (1). To this end, we construct a Landau-GinzburgDevonshire-type first-principles Hamiltonian by expanding the energy around the reference cubic phase of $P m \overline{3} m$ symmetry. The Hamiltonian includes the most important degrees of freedom of the structure: the strain $s_{i j}$, the tilts of the oxygen octahedra $q_{i}$, where $q_{i}$ represents the displacements of the oxygen atoms perpendicular to the rotation axis

TABLE II. Effective flexocoupling coefficients (absolute values in eV units) for (100)-, (110)-, and (111)-oriented samples in the beam-bending limit.

\begin{tabular}{llrr}
\hline \hline & $f_{\text {eff }}^{100}$ & $f_{\text {eff }}^{110}$ & $f_{\text {eff }}^{111}$ \\
\hline $\mathrm{LiOsO}_{3}$ & 40.1 & 5.3 & 2.5 \\
$\mathrm{BaTiO}_{3}$ & 16.2 & 24.7 & 23.3 \\
\hline \hline
\end{tabular}


and the polar modes $u_{i}$. (Details on the model can be found in Ref. [33], Secs. II and III.) First, we validate our effective Hamiltonian $H_{\text {eff }}$ by calculating the energetics of the relevant phases (Table S2) and their variation as a function of external pressure (Fig. S2); in both cases we obtain excellent agreement to the first-principles results. Next, we proceed to calculate the most favorable switching paths by constraining one component of the polar vector [46-48] and numerically minimizing (simulated annealing) the energy functional with respect to the other parameters.

The resulting double-well potential curves of $\mathrm{LiOsO}_{3}$ and $\mathrm{BaTiO}_{3}$ are shown in Fig. 2. Before commenting on the results, it is useful to recall the structural properties of each of the two materials. The structural ground state of $\mathrm{LiOsO}_{3}$ has $R 3 c$ symmetry, containing both polar distortions and antiphase octahedral tilts $\left(a^{-} a^{-} a^{-}\right.$in Glazer notation) oriented along the [111] pseudocubic direction. Since the
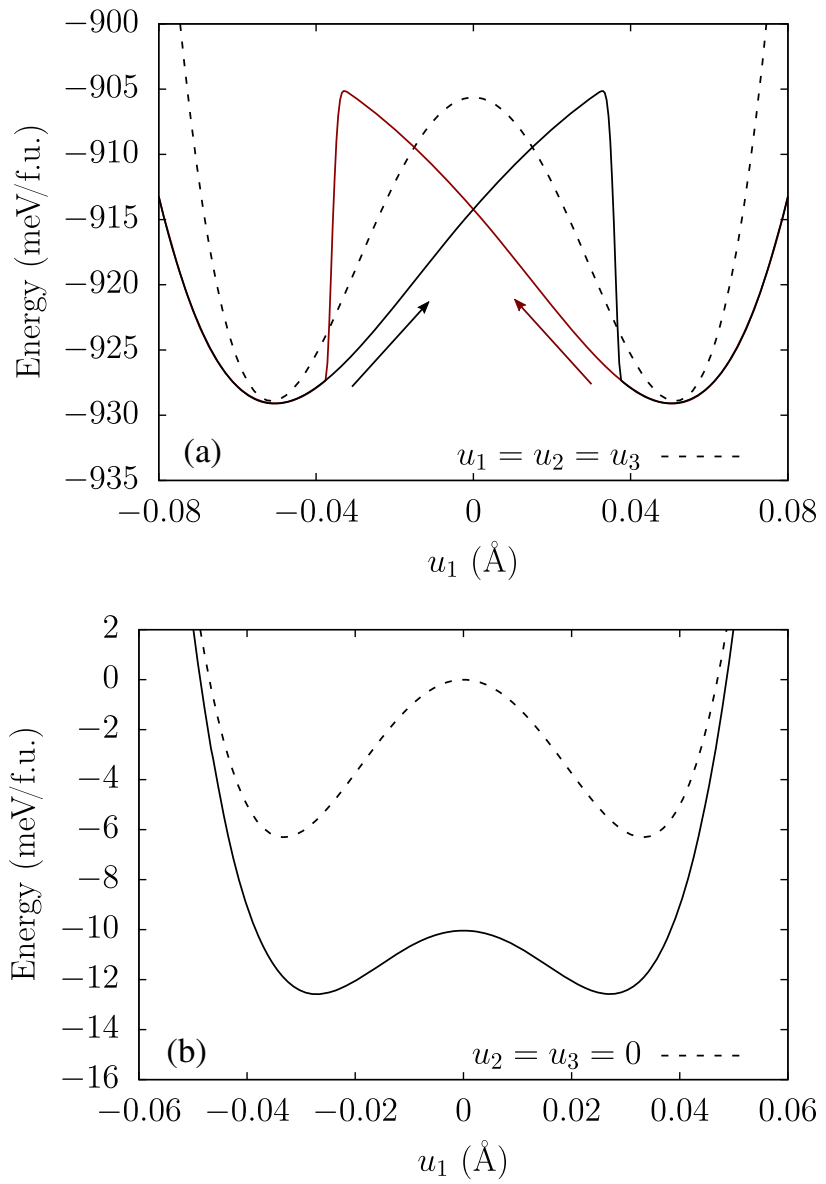

FIG. 2. Potential energy landscape for $\mathrm{LiOsO}_{3}$ (a) and $\mathrm{BaTiO}_{3}$ (b) from our first-principles effective Hamiltonians, obtained by minimizing the energy at fixed $u_{1}$. For $\mathrm{LiOsO}_{3}$, a double-welllike curve is obtained when $u_{1}=u_{2}=u_{3}$ is enforced (dashed line) and a butterflylike diagram is obtained when all the parameters are allowed to evolve freely (solid line, colors, and arrows are used to illustrate the switching path). For $\mathrm{BaTiO}_{3}$, the dashed line represents the study under the $u_{2}=u_{3}=0$ constraint, and the solid line the case with no constraints. energy scale associated to the antiferrodistortive (AFD) tilts is an order of magnitude larger than that associated to $\mathbf{u}$, they are unlikely to be affected by a weak elastic field; in practice, $\mathbf{u}$ can only switch between the [111] and $\left[\begin{array}{lll}\overline{1} & \overline{1} & \overline{1}\end{array}\right]$ states. Regarding the actual switching path, two scenarios are in principle possible. If the nonpolar $R \overline{3} c$ structure were stable under the constraint $u_{1}=0$, the polar modes would be forced to evolve along the same pseudocubic [111] direction even under the action of a [100]-oriented external force. However, previous first-principles calculations have shown [49] that the $R \overline{3} c$ phase has more than one imaginary mode at $\Gamma$, which means that $R \overline{3} c$ is unlikely to be the saddle point. This suspicion is nicely confirmed by the results of our effective Hamiltonian: indeed, the "butterfly diagram" of Fig. 2 clearly reflects the presence of a switchable in-plane polarization at $u_{1}=0$; the resulting coercive field is $F_{\text {coerc }}=0.34 \mathrm{eV} / \AA$. (The evolution of $u_{2,3}$ as a function of $u_{1}$ is shown in Fig. $\mathrm{S} 1$ of Ref. [33].) To quantify how much the system gains by circumnavigating the energy barrier, we attempted the same computational experiment while imposing $u_{1}=u_{2}=u_{3}$ along the path; as expected, we obtain a substantially larger critical field of $F_{\text {coerc }}=0.69 \mathrm{eV} / \AA$, assuming that the field is still applied along [100].

For $\mathrm{BaTiO}_{3}$ the polarization cannot be constrained by the tilts, since the latter are absent in this material. At lowtemperature $\mathrm{BaTiO}_{3}$ has $R 3 \mathrm{~m}$ symmetry, and we find that the lowest switching barrier occurs when the polarization continuously rotates from [111] to [1111] by passing through an orthorhombic [110] saddle point. (The path is roughly oriented along [100]). We find a critical coercive field of $F_{\text {coerc }}=0.14 \mathrm{eV} / \AA$ for such a switching path. For comparison to room-temperature experiments, where $\mathrm{BaTiO}_{3}$ adopts a tetragonal structure, we also calculate the hypothetical barrier that one would obtain by constraining $\mathbf{P} \|[100]$ (i.e., by setting the in-plane components of $\mathbf{P}$ to zero). We find $F_{\text {coerc }}=0.29 \mathrm{eV} / \AA$. This is a substantially larger value than the aforementioned threshold for polarization rotation, in line with literature results.

We are now ready to answer the main physical question we asked ourselves at the beginning: how much do we need to bend a $\mathrm{LiOsO}_{3}$ sample to reverse its polar lattice distortion? By means of Eq. (1) we can compute the critical bending radius for both materials. The obtained values are $R_{\text {crit }} \sim 118 \AA$ for $\mathrm{LiOsO}_{3}$ and $R_{\text {crit }} \sim 125 \AA$ for rhombohedral $\mathrm{BaTiO}_{3}$ and $R_{\text {crit }} \sim 60 \AA$ for tetragonal $\mathrm{BaTiO}_{3}$. Remarkably, the calculated critical bending radius of $\mathrm{LiOsO}_{3}$ is twice as large as that of tetragonal $\mathrm{BaTiO}_{3}$, essentially matching the calculated value of rhombohedral $\mathrm{BaTiO}_{3}$. Since mechanical switching of polar domains in tetragonal barium titanate has already been experimentally achieved $[22,50]$ via strain gradients, our results indicate that this is very likely to be feasible in $\mathrm{LiOsO}_{3}$ as well.

Our results for $\mathrm{BaTiO}_{3}$ are in good agreement with the ones reported in Ref. [51] where a critical bending radius of 
$110 \AA$ was estimated. This is substantially smaller than the available experimental estimates (a value of $R_{\text {crit }} \sim 300 \AA$ was observed in $\mathrm{BaTiO}_{3}$ [50]). This is expected: theoretical estimations of coercive fields in ferroelectrics that are based on the homogeneous Landau potential are typically overestimated by 1 or 2 orders of magnitude [52]. Consideration of more realistic mechanisms (e.g., domain wall nucleation and motion) would drastically complicate our study, and bring us far from our main scope. We stress in any case that our underestimation of the critical bending radii compared to experiments should be ascribed to an overestimation of $F_{\text {crit }}$, while we regard our calculation of the flexocouplings as accurate. (The contribution of the oxygen octahedral tilt gradients to the flexocoupling in lithium osmate was neglected in this work. While certainly present, we consider it unlikely to qualitatively affect our conclusions.)

As an outlook, we hope that the results presented here will stimulate further experimental work to verify our predictions. Also, it will be interesting to estimate the magnitude of the flexocouplings in a broader range of polar metals and identify candidates where the effect is especially strong. Finally, from the point of view of the theory, developing the methodological tools to assess the impact of tilt gradients on the calculated coefficients is another topic that we regard as promising for future studies.

We acknowledge the support of Ministerio de Economia, Industria y Competitividad (MINECO-Spain) through Grants No. MAT2016-77100-C2-2-P, No. PID2019108573GB-C22 and Severo Ochoa FUNFUTURE center of excellence (CEX2019-000917-S); and of Generalitat de Catalunya (Grant No. 2017 SGR1506). This project has received funding from the European Research Council (ERC) under the European Union's Horizon 2020 research and innovation program (Grant Agreement No. 724529). Part of the calculations were performed at the Supercomputing Center of Galicia (CESGA).

[1] T. H. Kim, D. Puggioni, Y. Yuan, L. Xie, H. Zhou, N. Campbell, P. J. Ryan, Y. Choi, J. W. Kim, J. R. Patzner, S. Ryu, J. P. Podkaminer, J. Irwin, Y. Ma, C. J. Fennie, M. S. Rzchowski, X. Q. Pan, V. Gopalan, J. M. Rondinelli, and C. B. Eom, Polar metals by geometric design, Nature (London) 533, 68 (2016).

[2] P. W. Anderson and E. Blount, Symmetry Considerations on Martensitic Transformations: "Ferroelectric" Metals?, Phys. Rev. Lett. 14, 217 (1965).

[3] E. Bauer and M. Sigrist, Non-Centrosymmetric Superconductors: Introduction and Overview (Springer Science \& Business Media, New York, 2012), Vol. 847.

[4] S. Yip, Noncentrosymmetric superconductors, Annu. Rev. Condens. Matter Phys. 5, 15 (2014).

[5] C.-K. Lu and S. Yip, Spin current in topologically trivial and nontrivial noncentrosymmetric superconductors, Phys. Rev. B 82, 104501 (2010).
[6] Y. Shi, Y. Guo, X. Wang, A. J. Princep, D. Khalyavin, P. Manuel, Y. Michiue, A. Sato, K. Tsuda, S. Yu et al., A ferroelectric-like structural transition in a metal, Nat. Mater. 12, 1024 (2013).

[7] N. A. Benedek and T. Birol, "Ferroelectric" metals reexamined: fundamental mechanisms and design considerations for new materials, J. Mater. Chem. C 4, 4000 (2016).

[8] D. Puggioni and J. M. Rondinelli, Designing a robustly metallic noncenstrosymmetric ruthenate oxide with large thermopower anisotropy, Nat. Commun. 5, 3432 (2014).

[9] M. Meng, Z. Wang, A. Fathima, S. Ghosh, M. Saghayezhian, J. Taylor, R. Jin, Y. Zhu, S. T. Pantelides, J. Zhang et al., Interface-induced magnetic polar metal phase in complex oxides, Nat. Commun. 10, 5428 (2019).

[10] Y. Cao, Z. Wang, S. Y. Park, Y. Yuan, X. Liu, S. M. Nikitin, H. Akamatsu, M. Kareev, S. Middey, D. Meyers, P. Thompson, P. J. Ryan, P. Shafer, A. N'Diaye, E. Arenholz, V. Gopalan, Y. Zhu, K. M. Rabe, and J. Chakhalian, Artificial two-dimensional polar metal at room temperature, Nat. Commun. 9, 1547 (2018).

[11] S. Ghosh, A. Y. Borisevich, and S. T. Pantelides, Engineering an Insulating Ferroelectric Superlattice with a Tunable Band Gap from Metallic Components, Phys. Rev. Lett. 119, 177603 (2017).

[12] Y.-W. Fang and H. Chen, Design of a multifunctional polar metal via first-principles high-throughput structure screening, Commun. Mater. 1, 1 (2020).

[13] H. Padmanabhan, Y. Park, D. Puggioni, Y. Yuan, Y. Cao, L. Gasparov, Y. Shi, J. Chakhalian, J. M. Rondinelli, and V. Gopalan, Linear and nonlinear optical probe of the ferroelectric-like phase transition in a polar metal, $\mathrm{LiOsO}_{3}, \mathrm{Appl}$. Phys. Lett. 113, 122906 (2018).

[14] C. Ma and K. Jin, Design strategy for ferroelectric-based polar metals with dimensionality-tunable electronic states, Sci. Chin. Phys. Mech. Astron. 61, 97011 (2018).

[15] Z. Fei, W. Zhao, T. A. Palomaki, B. Sun, M. K. Miller, Z. Zhao, J. Yan, X. Xu, and D. H. Cobden, Ferroelectric switching of a two-dimensional metal, Nature (London) 560, 336 (2018).

[16] A. K. Tagantsev, Piezoelectricity and flexoelectricity in crystalline dielectrics, Phys. Rev. B 34, 5883 (1986).

[17] P. Zubko, G. Catalan, and A. K. Tagantsev, Flexoelectric effect in solids, Annu. Rev. Mater. Res. 43, 387 (2013).

[18] P. Yudin and A. Tagantsev, Fundamentals of flexoelectricity in solids, Nanotechnology 24, 432001 (2013).

[19] S. M. Kogan, Piezoelectric effect during inhomogeneous deformation and acoustic scattering of carriers in crystals, Sov. Phys. Solid State 5, 2069 (1964).

[20] F. Vasquez-Sancho, A. Abdollahi, D. Damjanovic, and G. Catalan, Flexoelectricity in bones, Adv. Mater. 30, 1705316 (2018).

[21] J. Narvaez, F. Vasquez-Sancho, and G. Catalan, Enhanced flexoelectric-like response in oxide semiconductors, Nature (London) 538, 219 (2016).

[22] H. Lu, C.-W. Bark, D. E. De Los Ojos, J. Alcala, C.-B. Eom, G. Catalan, and A. Gruverman, Mechanical writing of ferroelectric polarization, Science 336, 59 (2012).

[23] M. Royo and M. Stengel, First-Principles Theory of Spatial Dispersion: Dynamical Quadrupoles and Flexoelectricity, Phys. Rev. X 9, 021050 (2019). 
[24] R. Resta, Macroscopic polarization in crystalline dielectrics: The geometric phase approach, Rev. Mod. Phys. 66, 899 (1994).

[25] M. Stengel, Flexoelectricity from density-functional perturbation theory, Phys. Rev. B 88, 174106 (2013).

[26] M. Stengel and D. Vanderbilt, First-principles theory of flexoelectricity, in Flexoelectricity in Solids From Theory to Applications, edited by A. K. Tagantsev and P. V. Yudin (World Scientific, Singapore, 2016), Chap. 2, pp. 31-110.

[27] M. Stengel, Unified ab initio formulation of flexoelectricity and strain-gradient elasticity, Phys. Rev. B 93, 245107 (2016).

[28] C. E. Dreyer, M. Stengel, and D. Vanderbilt, Current-density implementation for calculating flexoelectric coefficients, Phys. Rev. B 98, 075153 (2018).

[29] A. Schiaffino, C. E. Dreyer, D. Vanderbilt, and M. Stengel, Metric wave approach to flexoelectricity within density functional perturbation theory, Phys. Rev. B 99, 085107 (2019).

[30] A. H. Romero et al., ABINIT: Overview and focus on selected capabilities, J. Chem. Phys. 152, 124102 (2020).

[31] S. Tinte, J. Íñiguez, K. M. Rabe, and D. Vanderbilt, Quantitative analysis of the first-principles effective Hamiltonian approach to ferroelectric perovskites, Phys. Rev. B 67, 064106 (2003).

[32] U. V. Waghmare and K. M. Rabe, $A b$ initio statistical mechanics of the ferroelectric phase transition in $\mathrm{PbTiO}_{3}$, Phys. Rev. B 55, 6161 (1997).

[33] See Supplemental Material at http://link.aps.org/ supplemental/10.1103/PhysRevLett.126.127601 for more information about theoretical derivations, computational details. and results, which includes Refs. [34-36].

[34] M. Van Setten, M. Giantomassi, E. Bousquet, M. J. Verstraete, D. R. Hamann, X. Gonze, and G.-M. Rignanese, The PSEUDODOJO: Training and grading a 85 element optimized norm-conserving pseudopotential table, Comput. Phys. Commun. 226, 39 (2018).

[35] D. R. Hamann, Optimized norm-conserving vanderbilt pseudopotentials, Phys. Rev. B 88, 085117 (2013).

[36] J. P. Perdew, K. Burke, and M. Ernzerhof, Generalized Gradient Approximation Made Simple, Phys. Rev. Lett. 77, 3865 (1996).

[37] M. Royo, K. R. Hahn, and M. Stengel, Using High Multipolar Orders to Reconstruct the Sound Velocity in Piezoelectrics from Lattice Dynamics, Phys. Rev. Lett. 125, 217602 (2020).
[38] A. Yurkov and A. Tagantsev, Strong surface effect on direct bulk flexoelectric response in solids, Appl. Phys. Lett. 108, 022904 (2016).

[39] M. Stengel, Microscopic response to inhomogeneous deformations in curvilinear coordinates, Nat. Commun. 4, 2693 (2013).

[40] M. Stengel, Surface control of flexoelectricity, Phys. Rev. B 90, 201112(R) (2014).

[41] M. Born and K. Huang, Dynamical Theory of Crystal Lattices (Oxford University Press, Oxford, England, 1954).

[42] D. R. Hamann, X. Wu, K. M. Rabe, and D. Vanderbilt, Metric tensor formulation of strain in density-functional perturbation theory, Phys. Rev. B 71, 035117 (2005).

[43] X. Gonze, B. Amadon, P.-M. Anglade, J.-M. Beuken, F. Bottin, P. Boulanger, F. Bruneval, D. Caliste, R. Caracas, M. Côté et al., ABINIT: First-principles approach to material and nanosystem properties, Comput. Phys. Commun. 180, 2582 (2009).

[44] X. Gonze, B. Amadon, G. Antonius, F. Arnardi, L. Baguet, J.M. Beuken, J. Bieder, F. Bottin, J. Bouchet, E. Bousquet et al., The ABINIT project: Impact, environment and recent developments, Comput. Phys. Commun. 248, 107042 (2020).

[45] J. Narvaez, S. Saremi, J. Hong, M. Stengel, and G. Catalan, Large Flexoelectric Anisotropy in Paraelectric Barium Titanate, Phys. Rev. Lett. 115, 037601 (2015).

[46] O. Diéguez and D. Vanderbilt, First-Principles Calculations for Insulators at Constant Polarization, Phys. Rev. Lett. 96, 056401 (2006).

[47] M. Stengel, N. A. Spaldin, and D. Vanderbilt, Electric displacement as the fundamental variable in electronicstructure calculations, Nat. Phys. 5, 304 (2009).

[48] M. Stengel and J. Íñiguez, Electrical phase diagram of bulk $\mathrm{BiFeO}_{3}$, Phys. Rev. B 92, 235148 (2015).

[49] H. Sim and B. G. Kim, First-principles study of octahedral tilting and ferroelectric-like transition in metallic $\mathrm{LiOsO}_{3}$, Phys. Rev. B 89, 201107(R) (2014).

[50] J. Očenášek, H. Lu, C. W. Bark, C.-B. Eom, J. Alcalá, G. Catalan, and A. Gruverman, Nanomechanics of flexoelectric switching, Phys. Rev. B 92, 035417 (2015).

[51] G. Li, X. Huang, J. Hu, and W. Zhang, Shear-strain gradient induced polarization reversal in ferroelectric $\mathrm{BaTiO}_{3}$ thin films: A first-principles total-energy study, Phys. Rev. B 95, 144111 (2017).

[52] V. Shelke, D. Mazumdar, G. Srinivasan, A. Kumar, S. Jesse, S. Kalinin, A. Baddorf, and A. Gupta, Reduced coercive field in $\mathrm{BiFeO}_{3}$ thin films through domain engineering, $\mathrm{Adv}$. Mater. 23, 669 (2011). 